\title{
ACC

\section{Percepción del adulto mayor sobre su interrelación familiar. Sector II del Distrito La Victoria-Chiclayo-2018}

\author{
Peña Soplapuco Delia ${ }^{1}$, Pizarro Coronado Luz Bella ${ }^{2}$, Constantino Facundo Francisca ${ }^{3}$
}

\begin{tabular}{l} 
INFORMACIÓN DEL ARTÍCULO \\
\hline Historia del artículo: \\
Recibido el 20 de septiembre de 2019 \\
Aceptado el 14 de noviembre de 2019 \\
\hline Palabras clave: \\
Percepción \\
Anciano \\
Interrelación \\
Familia
\end{tabular}

\section{RESUMEN}

La presente investigación es de tipo cualitativa con abordaje metodológico de estudio de caso el cual tuvo como objetivo describir, analizar y comprender la percepción del adulto mayor sobre su interrelación familiar. Sector II del distrito La Victoria-Chiclayo-2018. Los sujetos de investigación fueron 15 adultos mayores, y 15 familiares que viven con el adulto mayor, ambas muestras fueron determinadas por la técnica de saturación y redundancia, para la recolección de datos se utilizó la entrevista semiestructurada que fue validada por juicio de expertos. Estos datos se procesaron a través del análisis de contenido temático obteniéndose cuatro categorías: El adulto mayor y su relación familiar, Apoyo de los hijos como parte importante de la relación familiar, sintiéndose útil: Participación del adulto mayor en las actividades del hogar y Actuación del adulto mayor como consejero en la familia. En todo momento de la investigación se tuvo en cuenta los criterios de rigor éticos y científicos. Concluyendo que la mayoría de adultos mayores y familiares consideran a la relación familiar como buena salvo algunas excepciones y refieren que para que exista esta buena relación es importante que estén los elementos de afecto, comunicación y compañía los cuales deben estar entrelazados uno de los otros, también el apoyo de los hijos a los padres es importante, como el permitir que el adulto mayor realice actividades dentro del hogar y lo más relevante es que son considerados como consejeros y orientadores en el seno familiar.

\footnotetext{
'Licenciada de Enfermería en Puesto Salud Tranca Fanupe, Lambayeque, Chiclayo, Perú. Email: karito_ps@hotmail.com 
Perception of the elderly adult about their family interrelation. Sector II of the District La Victoria-Chiclayo-2018

ABSTRACT

Keywords:

Perception

Elder

Interrelation

Family

The present investigation is of qualitative type with methodological approach of case study which had as objective to describe, analyze and understand the perception of the older adult about their family interrelation. Sector II of the district La Victoria-Chiclayo-2018. The research subjects were 15 older adults, and 15 relatives who lived with the older adult, both samples were determined by the technique of saturation and redundancy, for the data collection the semi-structured interview was used, which was validated by expert judgment. These data were processed through the analysis of thematic content, obtaining four categories: The elderly and their family relationship, Support of the children as an important part of the family relationship, Feeling useful: Participation of the elderly in the activities of the home and Action of the older adult as a counselor in the family. At all times of the investigation the criteria of ethical and scientific rigor were taken into account. Concluding that the majority of older adults and family members consider the family relationship to be good, except for a few exceptions, and mention that for this good relationship to exist, it is important that there are the elements of affection, communication and company which must be intertwined with one another, also the support of the children to the parents is important, such as allowing the older adult to carry out activities within the home and the most important thing is that they are considered as counselors and counselors within the family.

\section{Introducción}

La Organización Mundial de la Salud (OMS) menciona que la proporción de personas mayores se está extendiendo ligeramente en todo el mundo. Y, entre 2015 y 2050 esta proporción casi se duplicará, atravesando de 12 a 22\%, el crecimiento previsto es de 900 millones a 2000 millones de personas mayores de 60 años ${ }^{1}$. En Perú la población adulta mayor muestra un incremento considerable en la estructura de la pirámide en relación a la población infantil, en el año 1950 representaba el 5,7\% mientras que en el año 2016 fue de 9,9\%, al año 2017 tenemos una población adulto mayor de $10,1 \%^{2}$. A nivel regional el departamento de Lambayeque cuenta con una población de 1, 260,650 vecindarios, donde el $10,6 \%$ constituye la población adulto mayor ${ }^{3}$.

Este aumento conlleva a preocuparse por las personas de este grupo etáreo, debido a que el envejecimiento sufre modificaciones, tal es así que en lo físico, la piel se hace más delgada y seca, salen las canas, disminuye la fuerza muscular al igual que la capacidad de movimiento debido a la pérdida de calcio en los huesos ${ }^{4}$; en lo psicológico se evidencia cambios de los sentimientos y emociones caracterizado por acontecimientos traumáticos muy importantes $y$ continuados a lo largo de su etapa de la vejez, ya 
sea por la muerte de un ser querido, cambio de domicilio o por la pérdida de la actividad laboral, por otro lado existen una serie de parámetros por los que se da valor a la persona en su grupo social, los cuales se resumen en capacidad de trabajo y poder adquisitivo, a este fragmento poblacional anciana se sitúa entre los no productivos ${ }^{5}$.

Esto se evidenció en los resultados del estudio realizado por Barboza en el Hospital Distrital Santa Isabel en el 2016 sobre el funcionamiento familiar y el nivel de depresión del adulto mayor, con metodología de tipo cuantitativocorrelacional, la recolección de los datos se realizó mediante dos encuestas, la primera fue el Apgar familiar que consta de 5 interrogantes, que accedió valorar el nivel de funcionamiento familiar; también se utilizó la escala de Yesavage para establecer el nivel de depresión en el adulto mayor. Los efectos conseguidos fueron que el nivel normofuncional hubo relación con el estado anímico solo en un $32 \%$. Por lo tanto, existe relación entre el Nivel de Funcionamiento Familiar y el Nivel de Depresión en el Adulto Mayor del Hospital Distrital Santa Isabel El Porvenir- $2016^{6}$. Por ello es inevitable el apoyo familiar como un soporte vital basado en la comprensión, apoyo mutuo, comunicación, amor y solidaridad pues si no existiera esto pueden presentarse etapas de depresión pudiendo ser prolongados, es aquí donde la familia juega un papel muy significativo dentro de las relaciones interdependientes; pero llega un momento en que estos familiares deben continuar con sus obligaciones por trabajo, estudios, quehaceres de la vida etc., dejando al adulto mayor solo y con mucha responsabilidad, como el cuidado de los nietos y las tareas en el hogar, y no se percata que el adulto mayor necesita también de su atención y cuidado, dejando al olvido las conversaciones, los abrazos, y cariños acostumbrados.

La presente investigación nació como resultado de la observación y de la interacción realizada con los adultos mayores pertenecientes al grupo "María Jesús" del sector II de la Victoria durante las prácticas de pregrado, al dialogar con ellos se observó fascie triste, pensativos y algunos llorando al momento del conversatorio manifestando "señorita en mi casa me dejan al cuidado de mis nietos todo el día yo debo llevarlos al colegio, cocinarles" "mi hija llega tarde del trabajo y cuando llega no lava ni un plato" "dice que está cansada" "no ve a mis nietos" "no me pregunta si amanecíbien", "casi no hablamos" "me gustaría que esto termine", también referían "señorita vivo con una sobrina, ella me ha dejado solo en casa" "se ha ido, no sé cuándo regresará" "salgo de mi casa a pedir plata para comprar mi comida" "yo ya no trabajo" "señorita al estar aquí en el grupo me distraigo y me olvido un poco de mis problemas".

Esto conllevó a que las investigadoras se cuestionen lo siguiente $¿$ Es importante que exista una buena interrelación en las personas adultas mayores y su familia?, ¿La familia debe estar pendiente del adulto mayor? ¿El adulto mayor debe ser responsable de algunas actividades en el hogar? Luego de este interrogamiento nació la sucesiva interrogante de investigación: ¿Cómo es la percepción del adulto mayor sobre su interrelación familiar. Sector II del distrito la Victoria-Chiclayo-2018? Cuyo objetivo fue describir, analizar y comprender la percepción del adulto mayor sobre su interrelación familiar. Sector II del distrito la Victoria-Chiclayo-2018. El objeto de estudio fue Percepción del adulto mayor sobre su interrelación familiar.

Esta investigación se justifica debido a que la familia es estimada como el núcleo esencial de la sociedad quien tiene el compromiso del cuidado al adulto mayor, siendo esto muy significativo para conservar un envejecimiento saludable $y$ activo, puesto que debe encargarse de proporcionarle un ambiente cálido y de armonía para fortalecer su salud física, psíquica y emocional. De tal manera que en una Encuesta Nacional de Hogares (ENAHO) del año 2014, el $39,1 \%$ de los domicilios lo conforma algún adulto mayor de 60 y más años de edad y en el $8,1 \%$ de las viviendas vive alguno de 80 y más años de edad $^{7}$. 
Asimismo, permitió conocer la realidad que vive el adulto mayor dentro del seno familiar, y así llevar a una reflexión a la familia sobre la situación que pasan los adultos mayores, con el propósito de integrarlos. De esta manera servirá de punto de partida para posteriores investigaciones de acuerdo al objeto de estudio. También los resultados se darán a conocer a la institución correspondiente para que se tome las estrategias adecuadas con el fin de mejorar la relación del adulto mayor con su familia.

\section{Metodología}

Este estudio se basó en la investigación cualitativa $^{8,9}$ ya que nos permitió profundizar la percepción que tiene el adulto mayor sobre su interrelación familiar, así de esta forma describir los fenómenos que pudieron presentarse, buscando entender la realidad en su argumento natural tal y como sucedió, se abordó la metodología estudio de caso $^{9}$ método que permitió estudiar a fondo la percepción del adulto mayor sobre su interrelación familiar.

Los sujetos de investigación fueron 15 adultos mayores cuyas edades estuvieron comprendidas entre los 60 y 80 años de edad, fueron de sexo femenino y pertenecían al Centro de Salud María Jesús la Victoria-sector II, además cumplieron con los criterios de inclusión: Ser adultos mayores que libremente colaboraron con la investigación previo aceptación del consentimiento informado Ser activos y saludables, o con alguna enfermedad crónica no invalidante, la mayoría de los sujetos de investigación presentaban enfermedades como: hipertensión arterial y diabetes controladas. Además, estuvieron presente 15 familiares que vivían con el adulto mayor.

En el presente estudio se tuvo como primer escenario el Centro de Salud María Jesús el cual pertenece al Sector II del Distrito La Victoria, dicho establecimiento de salud se encontró ubicado en la calle Paul Harris $\mathrm{N}^{\circ} 119$, pertenece al primer nivel de atención de salud del Ministerio de salud (MINSA), con la categoría I-3, con servicio del programa del Adulto Mayor atiende a una población asignada de 2083 personas, aquí se captó a los participantes, del mismo modo se tuvo como segundo escenario la vivienda de cada adulto mayor ubicada en el distrito de La Victoria Sector II, las entrevistas se plasmaron en la sala de la vivienda de cada adulto mayor, posteriormente se procedió a entrevistar al familiar una vez terminada la entrevista al adulto mayor.

Para la recogida de datos se utilizó la técnica de la entrevista semiestructurada ${ }^{9}$. Y como instrumento se utilizó la guía de entrevista.

En primer lugar, se registró el proyecto de investigación en el catálogo de tesis de la escuela de enfermería y con el visto bueno de la docente de la asignatura de tesis I, se mostró al comité metodológico de dicha escuela para las sugerencias del caso; inmediatamente se presentó un ejemplar al Comité de Ética en Investigación de la Facultad de Medicina para su aprobación. Una vez aprobado el proyecto se procedió a solicitar el permiso respectivo a la Gerencia del Centro de Salud María Jesús - La Victoria. Posteriormente se hizo un análisis profundo de la realidad en el escenario establecido, con el fin de contactarse con los Adultos Mayores que efectúen con los criterios de inclusión, estableciendo con ellos la fecha y hora para la entrevista; previamente se les enseñó el consentimiento informado y se hizo firmar.

Después de la obtención de los datos se procedió a analizarlos por lo que se empleó el análisis de contenido temático, que permitió que se revelen temas y conceptos entre los datos recogidos, a medida que se avanza en el análisis de los datos, esos temas y concepciones se relacionan en una explicación más amplia. Este proceso presenta las siguientes fases ${ }^{10}$. Pre análisis, Codificación y Categorización.

En esta investigación se tuvo en cuenta los criterios de rigor científico de Hernández ${ }^{8}$. Credibilidad, Confirmabilidad, transferibilidad, además se tuvo en cuenta los principios éticos personalistas de Sgrecia ${ }^{11}$. Principio de respeto a 
la vida humana, Principio de libertad y responsabilidad, Principio de la sociabilidad y subsidiaridad.

\section{Resultados, análisis y discusión}

En el procesamiento de las entrevistas realizadas al adulto mayor y a sus familiares, se obtuvo las siguientes categorías:

I. El adulto mayor y su relación familiar

II. Apoyo de los hijos como parte importante de la relación familiar

III. Sintiéndose Útil: Participación del adulto mayor en las actividades del hogar

IV. Actuación del adulto mayor como consejero en la familia

Categoría I: El adulto mayor y su relación familiar

Para el adulto mayor es importante la relación con los otros, debido a que el hombre es un ser social por naturaleza y si deja de relacionarse, se aísla y se empobrece12; Es así que la afectividad que cada persona tiene es de especial importancia para el propio proceso de maduración y para las posibilidades de desarrollo como persona en un determinado contexto social, en las personas adultas mayores el área afectiva es otro de los elementos claves a tener en cuenta en la relación con los ancianos que soportan las consecuencias de la longevidad de su propio grupo ${ }^{5}$, el amor no es una cualidad regalo o virtud, sino un fenómeno biológico relacional básico y es la emoción que constituye la existencia social13. Esto se muestra en los siguientes discursos brindados por los adultos mayores que a continuación se presenta:

"...Mis hijas me tienen mucho afecto y yo también a ellas, me preocupo y trato de estar ayudando en lo que pueda..." A.MVerde

“...A mi hijo lo quiero mucho, todos los días nos abrazamos nos besamos y 10 apapacho..."A.M- Celeste
“...Yo abrazo bastante a mi nieto y él

también me abraza, así me siento contenta..." A.M- Crema.

Los discursos brindados por los adultos mayores y los familiares nos relatan que la relación en familia se basa en brindar afecto y amor a través de abrazos, besos, palabras, interrogantes que le ayudan a saber cómo está su familia dentro del hogar. Estos sentimientos enriquecedores hacen que los adultos mayores se sienten más seguros y protegidas, debido a que en esta etapa ocurren acontecimientos importantes necesitando sobre todo el apoyo de los hijos y nietos.

Por eso al enunciar afecto y brindar protección es fundamental en este grupo de personas, los vínculos apropiados entre padres e hijos deben estar basados en el cariño, los lazos fraternales y la relación de pareja, esto hace que los vínculos de parentesco sean más sólidas y se cimenten en afectos positivos, ya que quien no aprenda a querer en su casa, dificultosamente lo hará fuera de ella, salvo en situaciones excepcionales ${ }^{14}$.

Así como existen familias cuyo entorno está lleno de muestras de amor y cariño, en esta investigación se pudo evidenciar que en algunas familias ocurre lo contrario, tal como se evidencia en los siguientes discursos:

“...mis hijas no quieren que las abrace, ellas no me dan cariño, ni yo tampoco..." A.MCrema

“...Yo no soy de estar abrazándolos a cada rato a mis hijos, a veces les digo que los quiero..." A.M-Lila.

“...No le demuestro cariño a mi madre, pero en el fondo de mi corazón si la quiero..." F-

\section{Azucena}

Los adultos mayores mencionan que no les demuestran a sus hijos afecto a través de gestos o palabras a pesar de que tienen esos sentimientos no pueden exteriorizarlos pues los hijos al igual tampoco lo hacen, esto hace que algunas veces pueda interferir en el desarrollo de una adecuada relación familiar. En la familia el no poder expresar sentimientos de afecto hacia los demás puede 
darse debido a que ellos siguen culturas que han adquirido en el principio de su vida, las expresiones de amor son las que deben aparecer y transmitirse en primer lugar en el hogar ayudando a fortalecer sus relaciones.

A través de la comunicación y de la solidaridad, la familia fortifica el amor al otro y viceversa, así también hoy en la actualidad existen diferentes medios que ayudan a comunicar a pesar de la distancia, y con ellas establecer relaciones como llamadas telefónicas que permiten estar en constante interrelación y que siga presente ese cariño propio de una convivencia, desde luego que conservar este tipo de relaciones con personas que viven alejadas presume mayor esfuerzo, un pequeño abandono puede dañar delicadamente las pequeñas relaciones ${ }^{15}$.

La comunicación en la familia es uno de los pilares que fortalecen las relaciones y hacen que crezcan el amor, la comprensión y la confianza entre los miembros. Así como lo manifiestan los siguientes discursos.

“...Si nos comunicamos, converso con mis hijas y les pregunto cómo están mis nietos..."A.M-Verde.

“...Todos los días nos comunicamos, porque todos vivimos aquí, desde que Dios amanece hasta que Dios anochece, yo les cuento lo de la posta y ellos también se ríen conmigo y se alegran..." A.M-Blanco

"...Con ella converso todos los días me cuenta sus cosas como le ha ido en la iglesia, o con su hermana..." F-Orquídea.

Por medio de estos discursos se observa que la comunicación se conserva dentro del seno familiar mencionan que conversan con sus hijos todos los días sobre diferentes temas, les cuentan lo que les sucede cuando salen de casa, manteniéndolos unidos a través de las relaciones personales. La buena comunicación donde se escucha a la persona y se es escuchado es otra forma de fortalecer los lazos familiares, más aún los adultos mayores necesitan sentirse comprendidos al dialogar muestran sentimientos de satisfacción y valía personal.
Pero no en todas las familias se conserva una buena comunicación y esto debido a algunos factores como los malos entendidos, el quehacer diario, el trabajo, el individualismo de cada miembro, poca paciencia del familiar hacia el adulto mayor, así como también el autoritarismo de los hijos, dejando de lado las ideas que puede aportar el adulto mayor dentro del hogar, causando un inadecuado clima familiar tal como lo menciona el siguiente discurso:

“...lo que me pasa en el grupo del adulto mayor, no lo converso con mis hijos, porque no me escuchan, solo converso con mis amigas (Sra. Llora) ..." A.M-Crema.

Al hablar de convivencia se refiere a la proximidad física de las personas, se convive con las personas cercanas a nosotros, pero nuestro mundo de relación no se circunscribe exclusivamente a los que están a nuestro alrededor, también personas alejadas físicamente de nosotros forman parte de nuestra convivencia ${ }^{15}$. Tal es que en los siguientes discursos encontramos:

“...mis nietecitos vienen a visitarme, vienen dejando un día, ya no me olvidan..." A.MAmarillo

“...para ir al hospital, me acompañan mi nieta, a veces mi hija o hijo..." A.M-Celeste.

“...todos los domingos nos vamos a la misa, se siente alegre trato de que no se sienta mal por lo que falleció mi abuelita..." FDalia

Los adultos mayores manifiestan sentirse acompañados por su familia durante las diferentes actividades que deben realizar, lo cual les causa alegría y les hace sentirse bien. Villalobos en su estudio refiere: la presencia de otro que apoye al adulto mayor resulta crítico para que éste no se aísle ni se sienta solitario, lo cual le admitirá conservar un rol activo dentro del medio para así seguir desarrollándose, la compañía de los otros también le accederá saber que está rodeado por personas en las que puede confiar y con las que puede contar en caso de tener algún problema, 
todo esto le brindará seguridad e incluso podrá aumentar su tranquilidad y bienestar 16 .

Existen dentro de la población en estudio adultos mayores que expresan a través de sus discursos la necesidad de que alguien le acompañe en el día a día, podemos evidenciar que debido a las actividades que se presentan en su familia como el trabajar, atender a los hijos les resulta difícil poder compartir mucho más tiempo con ellos, tal como se expone en los siguientes discursos:

“...a veces estoy solita acá en mi cuarto, porque ellos se van a su trabajo, salgo a la calle, no me acompaña, me voy sola, solo me dan para mi pasaje ida y vuelta..." A.MRosado.

“...no tengo tanto tiempo de dedicar a mi madre porque no paro acá, pues mayormente paro en la calle, pero el poco tiempo que tengo me dedico a ella, ella frecuenta ir a la iglesia y la acompañamos..." F-Rosa.

En estos discursos las personas refieren que se encuentran solas y que no están acompañadas debido a que los familiares tienen que trabajar sintiéndose muchas veces solas, abandonadas y angustiadas por no tener a nadie con quien compartir su vida. Las personas no estamos hechas para estar solas, nuestro deseo de convivir no es fruto de un capricho, sino la exigencia de una necesidad, por eso el hombre solo enferma ${ }^{17}$. Por otro lado, podemos observar en el discurso siguiente el sentir de una adulta mayor que a pesar de vivir con sus hijos en algunos momentos de su vida siente la soledad y tristeza, así como lo manifiesta.

Categoría II: El apoyo de los hijos como parte importante de la relación familiar

El apoyo social es entendido como el conjunto de relaciones interpersonales que implica afecto y ayuda emocional (sentirse querido, intimidad, confianza, disponibilidad) instrumental (ayudas domésticas, cuidados) e informacional (búsqueda de información y consejos ${ }^{18}$. El apoyo que brinda la familia al adulto mayor es invalorable, el cual es ofrecido de diferentes maneras, desde el apoyo psicológico hasta el cuidado, incluyendo desde su salud hasta lo necesario para subsistir, estando así al tanto de todas las necesidades que se presentan, el apoyo económico en esta etapa es muy importante debido a que el adulto mayor se encuentra en una fase no productiva, y este apoyo brindado por parte de los hijos es invaluable. Los siguientes discursos nos muestran las diferentes formas como los familiares los apoyan a los adultos mayores para poder subsistir.

“...me compran mi comida, mi ropa, se preocupan por mi salud y me dan mi alimentación..." A.M-Turquesa.

"...me dan las cosas que necesito, mi ropa, medicinas para mí y mi esposo, nos dan alimentación, fruta, algún regalito y también vitaminas..."A.M-Verde.

“..económicamente ellos me ayudan, me mandan mi plata, víveres, me compran mi ropa todo lo que ellos puedan me envían..." A.M-Celeste.

Los hijos demuestran a través del apoyo económico de alguna manera poder compensar a los padres de una forma desinteresadamente por el tiempo, cariño, atención y cuidados brindados por los adultos mayores en sus años mozos, buscando así poder hacer sentir al adulto mayor feliz, demostrándole cariño a través de estas manifestaciones y de darle a conocer lo importante que son en la vida de ellos.

Por lo anterior, la mayoría de quienes trabajan lo hacen a partir de la informalidad, realidad actual en sus biografías personales desde la juventud. Es así que para ellos las actividades que generalmente realizan: son la venta de fruta, ropa y accesorios, también la elaboración y venta de manualidades. Estos mayores hallan en su trabajo el único medio de ingreso para su sustento familiar, que desde la informalidad es bajo, sin embargo, esa es la única opción que poseen para mantenerse. Esto se ve evidenciado en los siguientes discursos. 
"... Yo trabajo con mi esposo medio día en el mercado, cuando mis hijos nos dan dinero yo me siento tranquila, pero no nos alcanza..." A.M-marfil.

“...Mis hijos me ven ahora, mi esposo y yo no trabajamos, ellos nos dan el alimento y ropa, mi esposo no tiene trabajo..." A.MVioleta.

Lo manifestado en estos discursos permiten tener un panorama de las insuficiencias que muestran los adultos mayores y como ellos deben ver la forma de cubrir sus gastos. Por lo visto la familia es el principal medio de asistencia para el adulto mayor siendo este un elemento muy significativo dentro de las relaciones interpersonales ya que es una de las formas de demostrar preocupación por el bienestar del otro, los adultos mayores así como necesitan asistencia de los familiares, ellos también se muestran prestos para poder realizar cualquier actividad en casa retribuyendo de esta forma lo que sus hijos hacen por ellos y a la vez les hace descubrir que estas actividades los hacen sentirse útiles llegando a la siguiente categoría.

Categoría III: Sintiéndose Útil: Participación del adulto mayor en las actividades del hogar

Los adultos mayores se encuentran en una etapa de cambios, donde la mayoría ha dejado la vida activa laboral y ha pasado a estar más tiempo en casa, es aquí donde ellos buscan darle un nuevo horizonte a su vida tratando de encontrar formas de distribuir su tiempo y sentirse útiles. Asimismo, deben realizar actividades de la vida diaria debido a que todo lo que no se usa se atrofia y se derrocha, por lo que hay que permanecer haciendo todo aquello que les gusta, asimismo no se puede consentir que los demás hagan las cosas que pueden hacer ellos. Se debe hacer todo lo posible para conservar la autonomía y una buena manera de estar activos es ejecutando las acciones cotidianas como: salir a comprar, preparar la comida, arreglar la casa, fregar la vajilla, leer el periódico y doblar la ropa ${ }^{19}$.
En los siguientes discursos se evidencia como los adultos mayores se sienten satisfechos realizando las actividades en el hogar:

“...yo limpio mi casa todos los días y cocino, porque me hace sentirme bien..." A.MTurquesa.

“...yo me quedo en casita todos los días a cocinar, regar mis plantas y limpiar porque mi hija que trabaja es la que me ve, por eso tengo que colaborar y todavía lo puedo hacer..."A.M-Blanco.

Así también los familiares a través de sus discursos muestran que el adulto mayor realiza algunas actividades en casa porque se sienten bien:

“...mi mamá me ayuda limpiando la casa, a veces lavando los platos yo lavo la ropa de mi mamá eso le hace sentirse bien..." FGeranio.

“...cuando hace sus manualidades del grupo acá en casa nos quedamos hasta tarde, ella me ayuda cocinando cuando me voy a trabajar, a veces cocino yo y ella está viendo su gallina porque eso le hace sentirse alegre..."F-Gladiolo.

Dentro del seno familiar deben ser valoradas las capacidades físicas del adulto mayor, asignándosele tareas de acuerdo a su edad, obteniendo de esta manera satisfacción en los quehaceres del hogar tal como relatan los adultos mayores en estudio, mencionando que les gusta cocinar, lavar, hacer actividad creando un ambiente agradable en la familia, lo cual es respaldado por los argumentos ofrecidos por los familiares.

Tal como narra el adulto mayor en estudio, refiriendo que queda solo al cuidado del nieto en casa y no realiza otra actividad cotidiana cumpliendo de esa manera una actividad productiva dentro de casa. Sin embargo, existen también familias donde el adulto mayor es abrumado con las tareas del hogar, siendo causa de esto los hijos, donde muchas veces piensan erradamente que ellos disponen de mayor tiempo libre en casa, aprovechándose de estos, 
olvidándose de este modo de la vida personal de los mayores, ellos necesitan tiempo de relajación, recreación, socialización, etc., y no pueden realizar exceso de tareas ya que no poseen las mismas fuerzas que en la juventud. En los siguientes discursos podemos evidenciar el malestar del adulto mayor frente al atareado ritmo de vida que les toca llevar.

"...hasta ahora le tengo que dar de comer a mi nietito tengo que servirle su almuerzo, verlo a veces me canso ya, le digo a mi hija pero no tiene tiempo..." A.M-Lila.

“...yo cocino a todos los que viven en mi casa, también les lavo la ropa, no me ayudan mis hijas trabajan y a una de ellas la han operado..." A.M-Crema.

El adulto mayor necesita ser alentado en realizar las actividades de la vida diaria para mantenerse activo, las mismas que debe realizarse de acuerdo a su edad y teniendo en cuenta el esfuerzo físico, haciendo de esta manera que el adulto mayor se sienta útil e importante, asimismo el adulto mayor debe ser respetado en su hogar designándolo muchas veces dentro de la familia como consejero a quien acude para la solución de algún problema que puede darse en la familia. Esto nos permite el nacimiento de la siguiente categoría.

Categoría IV: Actuación del adulto mayor como consejero en la familia

Hoy en día el adulto mayor es considerado como una fuente llena de experiencias, de ahí la importancia de que el adulto mayor este reinsertado en la sociedad como una persona que puede hacer y ser, más que en lo que pueden tener, pues tienen potencialidades que ayudan a la familia a integrarse y muchas veces se convierten en un asesor dentro del seno familiar ya que por su experiencia logran mantener buenas relaciones familiares 20 .

"... a mi nieto yo lo aconsejo como debe de portarse con los amigos, que debe estar con amigos que sean buenos y que no lo lleven por mal camino, el si me escucha..." A.MRojo.

“...a mis nietos les aconsejo que estudien, que aprovechen el tiempo..." A.M-Verde.

“...a mis hijos les aconsejo que vivan bien y que se comprendan si hay un mal entendido, a veces le riño a mi hijo le digo que no tome mucho $y$ que debe estar tranquilo en una fiesta..." A.M-Turquesa.

En los discursos brindados por los adultos mayores se observa que la gran mayoría de ellos actúan como consejeros dentro del seno familiar, ya que existe un intercambio de afecto y apoyo que ha ido evolucionando a lo largo de los años y que por su constante interrelación hace que los miembros más pequeños como son los nietos acudan por su orientación y consejos ${ }^{21}$.

El papel que cumplen hoy en día los adultos mayores en la sociedad es de mucha importancia ya que ellos son los pilares del hogar, debido a su experiencia conocen las necesidades de cada miembro de la familia, además ellos son los que transmiten a sus hijos buenos valores que les llevan por el camino del bien. Tal es así que el Ministerio de Desarrollo e Inclusión Social está dando realce a los Adultos mayores con la finalidad de valorar sus conocimientos como legado para nuevas generaciones 22 .

\section{Conclusiones}

1. En el estudio la mayoría de adultos mayores y familiares describen sobre la relación familiar considerándola como buena, ya que a través de los discursos manifiestan que existen elementos importantes que se encuentran entrelazados uno de los otros, tal es así que por medio de abrazos y besos se muestran afecto y cariño. también es muy importante el contacto físico en la persona, más aún si es dentro de la familia transmitiendo de esta forma confianza, seguridad, amor $y$ fortaleciendo de este modo sus vínculos afectivos, encontrándose que la compañía y la comunicación son pilares significativos 
dentro de una familia, la compañía ayuda a crear esa cercanía entre ellos y de esta manera ir conociéndose y estableciendo un ambiente de armonía, el acompañarse implica interesarse por la otra persona buscando lo que necesita o le sucede, asimismo la comunicación permite que puedan expresar sus sentimientos el cual es una forma de demostrar su preocupación por la otra persona. Sin embargo también se encontraron adultos mayores que mostraron tristeza y sollozos al compartir su percepción de la relación familiar, manifestando ser distante debido a que no les brindan el tiempo necesario, sin embargo el no tener cerca a sus familiares por cuestiones de trabajo hace que se sientan solos, tristes afectando de esta manera al adulto mayor, para que exista una buena relación deben estar vinculadas el afecto, la comunicación y la compañía para que el adulto mayor pueda hacer más llevadero los cambios que ocurren en esta etapa de vida.

2. El apoyo de los hijos a los padres es muy importante en esta etapa de vida debido a los acontecimientos que se presentan, en el estudio los hijos tratan de cubrir las necesidades que padecen los adultos mayores, ya sean económicas o materiales, y de esta manera demuestran interés por los mayores. siendo importante en la relación familiar ya que de alguna manera demuestra su preocupación por ellos.

3. El adulto mayor en el hogar ha creado espacio para realizar labores domésticas, así también como el cuidado de los nietos, de esta manera hacen que se sientan útiles, sin embargo, existen familias que sobrecargan al adulto mayor con las labores creando en ellos malestar no permitiendo de este modo que gocen de una vejez saludable.

4. Los adultos mayores en estos tiempos siguen siendo considerados como consejeros $y$ orientadores en el seno familiar, debido a las experiencias vividas en la sociedad, lo cual es considerado valioso para los hijos y nietos, esto conlleva a que el adulto mayor sea engrandecido dándole la importancia que merece, de esta manera también se fortalece las relaciones familiares.

\section{Bibliografía}

1. Organización Mundial de la Salud. La salud mental y el adulto mayor [Internet]. Organización Mundial de la Salud. [consultado 28 de agosto de 2017]Disponible en: http://www.who.int/mediacentre/factsheets/f s381/es/

2. Instituto Nacional de Estadística e Informática. Situación de la población adulta mayor [Internet]. Lima. Informe técnico 2. [consultado 2017 de agosto 28]. Disponible en: https://www.inei.gob.pe/media/MenuRecursi vo/boletines/02informe-técnicon02_adulto-mayor-ene-mar2017.pdf

3. Comisión multisectorial. estadística del PAM 2015 [Internet]. Lambayeque estadísticas. [actualizado 2015 , consultado 28 de agosto de 2017]. Disponible en: https://www.mimp.gob.pe/adultomayor/regi ones/Lambayeque2.html

4. Leyton Z, Gómez M. El cuidado singular durante el envejecimiento y la vejez. Trujillo: Edunt; 2012. 239p.

5. García M, Rodríguez C, Toronjo A. Enfermería geriátrica. Barcelona: ediciones Difusión avances de enfermería; 2012. 448p.

6. Barboza B. Funcionamiento familiar y su relación con el nivel de depresión del adulto mayor del hospital distrital Santa Isabel, El Porvenir-La Libertad 2016. [Tesis de grado en Internet]. Trujillo: Universidad César Vallejo, 2016. [consultado 6 de octubre 2017]. Disponible en:

http://alicia.concytec.gob.pe/vufind/Record/ UCVV_fd7b882ede34e48fe36cab4 040b75dc7

7. Instituto Nacional de Estadística e Informática. 
En el Perú viven más de 3 millones de adultos mayores [Internet]. Lima. [consultado 26 de noviembre 2017].Disponible en: https://www.inei.gob.pe/prensa/noticias/enel-peru-vivenmas-de-3-millones-deadultos-mayores-8570/

8. Hernández R, Fernández C, Baptista $M$. Metodología de la investigación. 6ed. México 2014. P. 599.

9. Cupani A, Leal A, Hernández M. Calidad de la investigación cualitativa en enfermería. En: Do Prado M, De Souza M, Monticelli M, Cometto M, Gómez P. Investigación cualitativa en Enfermería. Metodología y didáctica. Washington: 2013.p.3-11

10. De Souza M, Arzuága M, Gonzales Y. Metodología del análisis de contenido en investigación de enfermería. En: Do Prado $M$, De Souza M, Monticelli M, Cometto M, Gómez P. Investigación cualitativa en Enfermería. Metodología y didáctica. Washington: 2013. P100-107.

11.Sgreccia E. Manual de bioética. Madrid: BAC Maior; 2014.968p

12. Caballero F. La salud en la tercera edad. Madrid. Cultural S.A; 2006.224p.

13. Maturana H, Verden Z. Amor y juego, fundamentos olvidados de lo humano. Santiago de Chile: Instituto de Terapia Cognitiva, 1993.

14. Osoria A. Cobián A. Desarrollo del clima familiar afectivo y su impacto en el bienestar subjetivo de la familia. Multimed. [Internet]. 2015 [citado 18 de junio 2018] 19(2) Disponible en: file:///C:/Users/JOSE/Downloads/239-17031-PB.pdf

15. García M. La convivencia. 5 ed. Madrid. 2001. $124 \mathrm{P}$.
16. Villalobos E. Apoyo Social en los adultos mayores de un centro de salud de José Leonardo Ortiz-Chiclayo. [Tesis de Licenciatura]. Chiclayo: Universidad Católica Santo Toribio de Mogrovejo, 2014[Consultado el 20 de Junio]. Disponible en: http://tesis.usat.edu.pe/bitstream/usat/352/1 /TL_Villalobos_Cordova_Elda.pdf

17. Rivadeneira, J, López, M. Escala de comunicación familiar: validación en población adulta chilena. Acta Colombiana de Psicología. [Internet]. (2017). 20(2),116-126. Disponible en:

http://repository.ucatolica.edu.co/bitstream/ 10983/15300/1/Escala\%20de\%20C omunicaci\%C3\%B3n\%20Familiar.pdf

18. Soldevida A. Los centros de vida para personas mayores. España: Edicions de la universitat de Lleida; 2003. [citado 2018 Nov 16]. Disponible en:

https://books.google.com.pe/books?id=k2X3 Y5HckfgC\&pg =PA92\&dq =apoyo + de + los + hij os $+\mathrm{a}+$ los + padres + ancianos $\& \mathrm{hl}=\mathrm{es} 419 \& \mathrm{sa}=\mathrm{X}$ \&ved=0ahUKEwj2ybGM4K_gAhUuTt8KHZT8AJ IQ6AEISZAG\#v=onepage $\& \mathrm{q}=$ apoyo $\% 20 \mathrm{de} \% 20 \mathrm{I}$ os\%20hijos\%20a\%20los\%20padres\%20anciano $\underline{\mathrm{s} \& \mathrm{f}=\mathrm{false}}$

19. Perlado F. Teoría y práctica de geriatría. Madrid. Ediciones Díaz de Santos, S.A; $1995.313 P$

20. Gutierrez L. Los Adultos Mayores La Reserva de los Saberes. Quito [Internet].2013. [citado 2019 Ene 30]; 13(6). Disponible en: http://cemlad.edu.ec/wpcontent/uploads/2013/11/2013-Adultosmayores-reserva-de-los-saberes.pdf

21. Papalia D, Duskin R, Martorell G. Desarrollo humano.12 ed. Mexico: McGraw-Hill education; 2017.800p. 
22. Mines, M. Ministerio de Desarrollo e inclusión Social. Adultos Mayores compartieron sus saberes con nuevas Generaciones. [Internet]. agosto 20, 2018. [citado 12 de febrero de 2019] Disponible en: https://www.pension65.gob.pe/adultosmayores-de-carabayllo-compartieron-sussaberes-con 